\title{
INVERTIR EN ARTE: LA COLECCIÓN DE PINTURA REUNIDA POR EL COMERCIANTE ESTADOUNIDENSE RICHARD WORSAN MEADE EN CÁDIZ ENTRE 1804 Y 1820*
}

\author{
GuAdalupe CARRASCO ${ }^{1}$ \\ Universidad de Cádiz
}

\begin{abstract}
Las guerras napoleónicas fueron una oportunidad para algunos comerciantes estadounidenses para comerciar con España. El comerciante estadounidense Richard W. Meade se estableció en Cádiz entre 1804 y 1820. En estos años consiguió acumular una importante fortuna. Su estancia en Cádiz durante la invasión francesa le permitió reunir una valiosa colección de obras de arte, sobre todo pintura barroca española e italiana. A través de los catálogos de subastas de la colección publicados en 1831 y 1853 y de los manifiestos de carga de los barcos llegados a Filadelfia conservados en la Custom House se ha podido conocer casi en su totalidad la colección de pinturas que Richard W. Meade trasladó a Estados Unidos.
\end{abstract}

Palabras clave: Estados Unidos; Meade; Cádiz; Obras de arte.

\section{INVESTMENT IN ART: THE COLLECTION OF PAINTINGS ASSEMBLED BY THE AMERICAN MERCHANT RICHARD WORSAN MEADE IN CÁDIZ BETWEEN 1804 AND 1820}

The Napoleonic wars were an opportunity to trade with Spain for some American merchants. The American merchant Richard W. Meade was resided in Cádiz between 1804 and 1820. During those years Meade managed to accumulate an important fortune, which allowed him to unite a valuable collection of artworks, mainly comprised of Spanish and Italian Baroque paintings. Thanks to the auction catalogs of the Meade sales published in 1831 and 1853, plus the ships' manifests preserved in the Custom House of Philadelphia, it has been possible to ascertain-almost in its totality- the collection of paintings that he sent to the United States.

Key words: United States; Meade; Cádiz; artworks.

Cómo citar este artículo / Citation: Carrasco, Guadalupe (2019): "Invertir en arte: la colección de pintura reunida por el comerciante estadounidense Richard Worsan Meade en Cádiz entre 1804 y 1820”. En: Archivo Español de Arte, vol. 92, núm. 365, Madrid, pp. 65-82. https://doi.org/10.3989/aearte.2019.06.

\section{Introducción}

El comercio marítimo colonial convirtió a Cádiz en el puerto de mayor tráfico comercial de España desde 1679, cuando se trasladó allí la cabecera de las flotas de Indias. A lo largo del siglo XVIII los cambios legislativos y administrativos aplicados por la nueva dinastía borbónica con-

\footnotetext{
* Esta investigación fue realizada en 2016 durante una estancia como fellowship en Filadelfia en The Historical Society of Pennsylvania y en The Library Company of Philadelphia.

${ }^{1}$ guadalupe.carrasco@uca.es / ORCID iD: http://orcid.org/0000-0002-5843-5811.
} 
solidaron su papel como puerto del monopolio colonial español mientras que su población crecía al compás de los beneficios de los comerciantes que llegaban incesantemente del resto de España y de otros estados europeos, alcanzando en 179177.500 habitantes 2.

En la última década del siglo XVIII y los años iniciales del siglo XIX la prosperidad del comercio se fue eclipsando a consecuencia de los conflictos recurrentes de España con Francia e Inglaterra. El comercio con América se vio interrumpido obligando a la monarquía a autorizar, en 1797, la intervención de los estados neutrales y señaladamente de Estados Unidos como intermediarios necesarios en el comercio colonial. Sin embargo, la oposición airada de los comerciantes gaditanos obligó a la revocación de dicha autorización en 1799 y a sustituirla por un sistema de licencias.

Al mismo tiempo, al otro lado del Atlántico las Trece Colonias convertidas en la nueva república de Estados Unidos intentarán construir su economía a partir de los principios mercantilistas que ponían énfasis en el comercio exterior. Para Estados Unidos las guerras revolucionarias y napoleónicas supusieron una oportunidad para su flota mercante que, aprovechando su neutralidad, creció de manera constante entre 1790 y 1815. La presencia estadounidense, a partir de entonces, en los puertos europeos precisó de una estructura diplomática, que en el caso de España se materializó en la construcción de una red de oficinas consulares en los puertos más impor$\operatorname{tantes}^{3}$ y en la firma de un tratado de paz, amistad y comercio en 1795.

La progresiva importancia del puerto de Cádiz para el comercio estadounidense se pone de manifiesto en el incremento constante durante el periodo señalado de la llegada de buques de aquella nación ${ }^{4}$. Esta realidad choca con el escaso número de comerciantes estadounidenses que se instalaron en la ciudad, a pesar de que la oficina consular de Cádiz fue una de las primeras en abrirse en España. Entre ellos destaca Richard W. Meade que llegó a Cádiz, procedente de Filadelfia en 1804 y permaneció en España hasta su regreso en 1820. Meade amasó una considerable fortuna, que no solo reinvirtió en sus negocios sino que la empleó en vivir en Cádiz de manera acomodada, alquilando una casa en el mejor barrio de la ciudad, amueblándola con todo tipo de comodidades y lujo y decorándola con una valiosa colección de pinturas 5 .

Así pues, en este artículo analizaré las circunstancias que rodearon la constitución de la colección artística que logró reunir Richard W. Meade durante su estancia en Cádiz, cuándo y cómo pudo adquirir estas obras, qué pinturas formaban parte de la colección, sus autores, sus precios y cuál fue su destino en Estados Unidos.

Las fuentes provienen de archivos y bibliotecas estadounidenses y son diversas. Fundamentalmente se trata de los dos catálogos publicados para la venta primero y la subasta, después, de parte de la colección, así como los manifiestos de carga, conservados en la Custom House de Filadelfia ${ }^{6}$, de los barcos estadounidenses que arribaron procedentes de Cádiz y en los que Richard W. Meade cargó los muebles, los libros y los cuadros. El estudio conjunto de estas fuentes puede ofrecer una perspectiva interesante del desarrollo del gusto por el arte español en Estados Unidos en las décadas iniciales del siglo XIX.

\section{Algunas consideraciones sobre el coleccionismo en Cádiz a finales del siglo XVIII}

El coleccionismo de obras de arte fue un fenómeno cultural que se desarrolló durante los siglos XVII y XVIII y que en este último siglo se llega a convertir en un medio de promoción y distinción social y cultural. Se trata de una actividad ligada casi de manera exclusiva a las clases privilegiadas (nobleza y clero), aunque también tiene cabida entre la burguesía, particularmente

\footnotetext{
2 Bustos, 2005: 79.

3 Carrasco-González, 2010: 234-247.

4 Carrasco-González, 2016: 177.

5 Carrasco-González, 2015.

6 Roe, 2010: 38.
} 
entre los mercaderes y hombres de negocios que mantenían relaciones comerciales con América y Europa, y entre los letrados y funcionarios que desempeñaban altos cargos en la administración.

En Sevilla y Cádiz el comercio con América favoreció la formación de grandes fortunas, buena parte de las cuales se invirtieron en bienes raíces, en el lujoso mobiliario de sus viviendas y en bibliotecas y colecciones de arte. Son bastante bien conocidos las colecciones artísticas sevillanas, especialmente las de las casas nobiliarias, las de algunos comerciantes y algunas pertenecientes a miembros del clero ${ }^{7}$. En Cádiz, sin embargo, la ausencia de particiones en la documentación notarial conservada, ha impedido el conocimiento más profundo del mobiliario, de las bibliotecas y de las obras de arte que a buen seguro guardaban muchas casas de Cádiz. No obstante, se tienen datos de algunos casos en los que los escasos inventarios de bienes han recogido descripciones del mobiliario, joyas y con menos frecuencia de bibliotecas y de obras arte. Se han analizado las bibliotecas de algunos miembros del clero, abogados ${ }^{8}$ y miembros de la Armada Real, pero también se conoce el interés de un corto número de comerciantes por los libros y las obras de arte. Algunos de ellos han sido objeto de profundos análisis como el caso de Sebastián Martínez Pérez ${ }^{9}$, Pedro Alonso O'Crowley ${ }^{10}$, el Conde de Maule ${ }^{11}$ y Juan Nicolás Böhl de Faber ${ }^{12}$ y con un menor poder adquisitivo Bernardo de Elías ${ }^{13}$.

Antonio Ponz constata en 1791, el interés de algunas "personas de buen gusto... por las colecciones de pinturas y otras curiosidades muy estimables"14. El propio Ponz visitó algunas de las casas de Cádiz donde había destacadas colecciones, citando expresamente la de Sebastián Martínez ${ }^{15}$ formada por al menos 300 pinturas, y de la que describe algunos cuadros notables, la de Pedro O'Crowley con "un par de centenares de piezas"16 y la de Joseph de Murcia "una colección de pinturas muy digna de cualquier curioso" 17 . Otro viajero, el estadounidense Mordecai Noah que pasó por Cádiz entre 1813-1814 señala con admiración la existencia de una Academia de Dibujo y Bellas Artes y la existencia de notables colecciones privadas de arte que permanecieron intactas gracias a que los franceses no llegaron a tomar la ciudad, pero indica que la mayoría son propiedad de los conventos e iglesias que hay en la ciudad ${ }^{18}$.

Si escasas son las noticias sobre el número y la calidad de las colecciones privadas, menos se sabe todavía sobre los distintos métodos utilizados en la adquisición de los libros y las obras de arte. Es conocida la tradición británica entre los miembros de la nobleza y la alta burguesía europea en el siglo XVIII de realizar un viaje como medio de formación integral. En Cádiz era también habitual entre los nobles y ricos comerciantes y sabemos que al menos, el conde de Maule, el marqués de Méritos, el marqués de Ureña, Francisco de Paula Iribarren, Juan Nicolás Böhl de Faber y Pedro Alonso O'Crowley viajaron por Europa ${ }^{19}$. Estos periplos que se recogían en diarios de viajes (handbooks) les permitía aprovisionarse de libros y obras de arte. Maule recuerda en su epistolario, las compras que fue haciendo en su viaje por Europa, y sabemos también que a su llegada a Cádiz el marqués de Ureña registró en la aduana varios cajones llenos de libros y pinturas ${ }^{20}$. La vida en una ciudad comercial y cosmopolita como Cádiz y su actividad comercial permitieron a estos potentados la adquisición de obras de arte en los distintos mercados europeos gracias a sus redes de correspondientes. Al mismo tiempo debió existir un mercado de

\footnotetext{
7 Antigüedad/Alzaga, 2011.

8 García González, 1999.

9 Pemán, 1978: 53-62, 1992: 303-320; García-Baquero, 1988.

10 Antón Solé, 1966: 151-165.

11 Ibáñez Vergara, 1997; Ravina, 1997.

12 Ravina, 2001: 89-102.

13 Gil-Díez, 2011: 31-47.

14 Ponz, 1794: 19-20.

15 Ponz, 1794: 20.

16 Ponz, 1794: 26.

17 Ponz, 1794: 27.

18 Mordecai, 1819: 140.

19 Ravina, 2001: 95.

20 Ravina, 2001: 95.
} 
obras de arte en las ciudades vinculadas al comercio de Indias como Sevilla, Sanlúcar, El Puerto de Santa María e incluso Jerez, donde nobles, altos funcionarios, cosecheros y comerciantes interesados por el arte compraban y vendían pinturas cambiando así de manos por diversas razones. Antonio Ponz cuenta como vio en casa de Sebastián Martínez "el borron de Murillo" para los Desposorios de Santa Catarina que había visto unos años antes en El Puerto de Santa María "en poder de otro dueño" 21 . El fallecimiento de uno de estos coleccionistas podía poner en circulación libros y obras de arte. Este fue el caso de al menos una parte de la colección de pinturas y estampas, de Sebastián Martínez cuyos bienes se dividieron entre sus dos hijas; y, según cuenta el conde de Maule, al menos una de sus hijas, Catalina, vendió las obras de arte que le correspondieron a los ingleses, sin dar más detalles sobre los compradores ${ }^{22}$. Pero también es admisible la hipótesis de que en los años finales del siglo XVIII y en la primera década del siglo XIX este mercado estuviese mejor abastecido, debido a las dificultades por las que atravesó el comercio gaditano con numerosas quiebras de importantes casas comerciales, a consecuencia de las gue$\operatorname{rras}^{23}$. Es posible que muchos comerciantes se vieran obligados a vender o entregar parte de su patrimonio para superar los malos tiempos. Además, la invasión francesa de la península contribuyó también a alimentar tanto el mercado gaditano de arte como el europeo, poniendo a disposición de coleccionistas privados muchas obras de arte procedentes del expolio realizado en monasterios, iglesias y palacios. Durante esos años algunos marchantes, particularmente británicos, acudieron solícitos a España para suministrar al mercado inglés pinturas españolas, cuya cotización hasta entonces estaba muy por debajo de la flamenca e italiana ${ }^{24}$. Algunos de estos mercaderes de arte, como James Wisseman, tenían buenos contactos entre los comerciantes sevillanos y gaditanos, por haber residido en Sevilla y pertenecer a una de las familias de comerciantes extranjeros de larga tradición comercial en esa ciudad y en Andalucía ${ }^{25}$.

El interés por reunir libros y obras de arte no respondía a una única motivación sino que era consecuencia de una combinación de razones que aunaban motivos filantrópicos junto a otros prácticos y espurios. La obra de arte no solo tenía valor decorativo, la posesión de mobiliario suntuoso, una imponente y hermosa biblioteca con vistosas encuadernaciones y numerosas obras de arte eran reflejo de poder y éxito en los negocios. Cuando Pedro O'Crowley pensaba en "edificar una galería para colocar dichas obras (pinturas) con buen orden" 26 es probable que considerara todas esas razones. Porque todo esto también formaba parte de la imagen que el comerciante pretendía proyectar. Su casa era el escaparate de su reputación y de su notoriedad, era el resultado tangible de los beneficios obtenidos en su actividad negociadora. En una vivienda llena de cosas hermosas un comerciante extranjero podía agasajar a sus compatriotas y posibles clientes mostrando así la conveniencia de hacer negocios con él. Es entonces probable que entre los fines de este interés por reunir libros, muebles y obras de arte estuviera también la de generar confianza y una reputación, demostrando ser un hombre cultivado y viajado, con contactos y conocedor de las últimas novedades.

Más allá del status social que proporcionaba la posesión de obras de arte, a principios del siglo XIX el arte empezó a convertirse en un bien que cotizaba al alza en el mercado, lo que significaba para sus poseedores una buena inversión, sobre todo para comerciantes que residían temporalmente en algunos puertos y ciudades y que cambiaban de residencia con cierta frecuencia. Las obras de arte se revalorizaban y eran un capital fácil de transportar y de convertir en dinero si se elegía bien el mercado donde colocarlas. Uno de esos mercados atractivos podía ser el estadounidense, donde una burguesía cultivada en ciudades como Filadelfia se había enriquecido rápidamente en la última década del siglo XVIII y era consumidora habitual de arte.

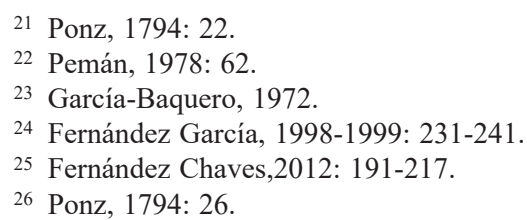




\section{El patrimonio mobiliario de Richard W. Meade resultado de sus negocios en Cádiz}

Richard W. Meade fue un comerciante estadounidense que residió en España entre $1804 \mathrm{y}$ 1820, en Madrid y en Cádiz, aunque fue en esta última ciudad donde estableció su residencia familiar y su casa comercial. La llegada de Richard W. Meade a España fue consecuencia de las difíciles relaciones diplomáticas con Estados Unidos a causa del embargo por parte de las autoridades españolas de un número considerable de barcos y mercancías estadounidenses en Montevideo y Buenos Aires, en 1802, cuyas cargas estaban valoradas en un millón de dólares ${ }^{27}$. Richard W. Meade fue elegido de entre la treintena de comerciantes estadounidenses afectados por el embargo, para negociar ante el gobierno español la reclamación de lo requisado, en el marco del convenio hispano-estadounidense firmado en 1802.

Cuando llega a Cádiz, Meade ya era un experimentado comerciante con una reputación y una considerable fortuna adquirida en el comercio con las Indias occidentales, Gran Bretaña y Francia. En Cádiz actuará como consignatario y agente de mercaderes de los principales puertos estadounidenses. Entre 1805 y 1816 recibió, en Cádiz, al menos 445 buques y entre 1809 y 1812 el $72 \%$ de los barcos estadounidenses que arribaron vinieron consignados a su nombre. Meade amasó una notable fortuna, en parte gracias a la necesidad de provisiones a causa de la invasión francesa de la península y la movilización de tropas españolas e inglesas. En este contexto se deben insertar los contratos de suministros que Meade firmó con la Junta Central y la Regencia ${ }^{28}$, que serían finalmente los responsables del largo contencioso que mantuvo con el estado español primero, y después con el gobierno estadounidense para reembolsarse el valor de las provisiones vendidas y que fueron también el origen de su ruina ${ }^{29}$.

El conocimiento y estudio del patrimonio de Richard W. Meade ha sido posible gracias al análisis de una serie de fuentes diversas y dispersas, pero muy valiosas por cuanto apoyan la hipótesis de la gran fortuna personal, la personalidad y las inclinaciones políticas de un comerciante estadounidense a principios del siglo XIX. Una parte importante de estas fuentes se encuentran en distintos archivos de su ciudad natal, Filadelfia, a la que regresó tras una estancia de cerca de veinte años en España. Allí se ha localizado su "última voluntad" redactada en Cádiz en $1820^{30}$, antes de zarpar con destino a Nueva York y el inventario elaborado por la Orphan's Court a su muerte en 1828, donde además del mobiliario y el ajuar, se recoge en una sola partida los 1.085 volúmenes de la biblioteca y 156 pinturas y esculturas repartidas en seis partidas $^{31}$.

El traslado de todos estos bienes desde Cádiz a Filadelfia se debió realizar en varios barcos, de los que he localizado dos ${ }^{32}$, uno en 1810 y otro en 1816, en cuyos manifiestos de carga figuran explícitamente algunos de los muebles del inventario, los títulos de 61 libros y varias cajas conteniendo pinturas. Además ha sido posible documentar una buena parte de las pinturas que Richard W. Meade trasladó a Estados Unidos a través de los catálogos de la exposición y la subasta de la colección que se realizaron en $1831^{33}$ y $1853^{34}$ (fig. 1).

El inventario valorado de los bienes de Meade no está organizado por partidas, sino que resulta ser una enumeración, en primer lugar de todo el mobiliario, donde se incluyen los instrumentos de música, muebles, libros, pinturas y esculturas, continuando con la plata, cristalería y vajillas de porcelana y china. Al final se recoge de manera detallada el vino, en cajas de 12 bo-

\footnotetext{
27 Coatswort, 1967: 244.

${ }^{28}$ Los contratos firmados ascendían a once millones de reales de vellón. Carrasco-González, 2015.

${ }^{29}$ La deuda pendiente con Meade ascendía a 378.879, 88 dólares, que nunca se pagaron. Carrasco-González, 2015 : 109-113

${ }^{30}$ Historical Society of Pennsylvania (HSP), Cadwalader Papers, Legal Papers, Box 13.

31 HSP, Cadwalader Papers, Legal Papers, Box 13.

32 Pudo haber otros que arribaran a otro puerto, bien a Nueva York o a Baltimore.

${ }^{33}$ Frick Art Reference Library, NY, The Frick Collection, ND454. M33, 1831. A.

${ }^{34}$ Library Company of Philadelphia, SM \#AM 1853, Mea.
} 


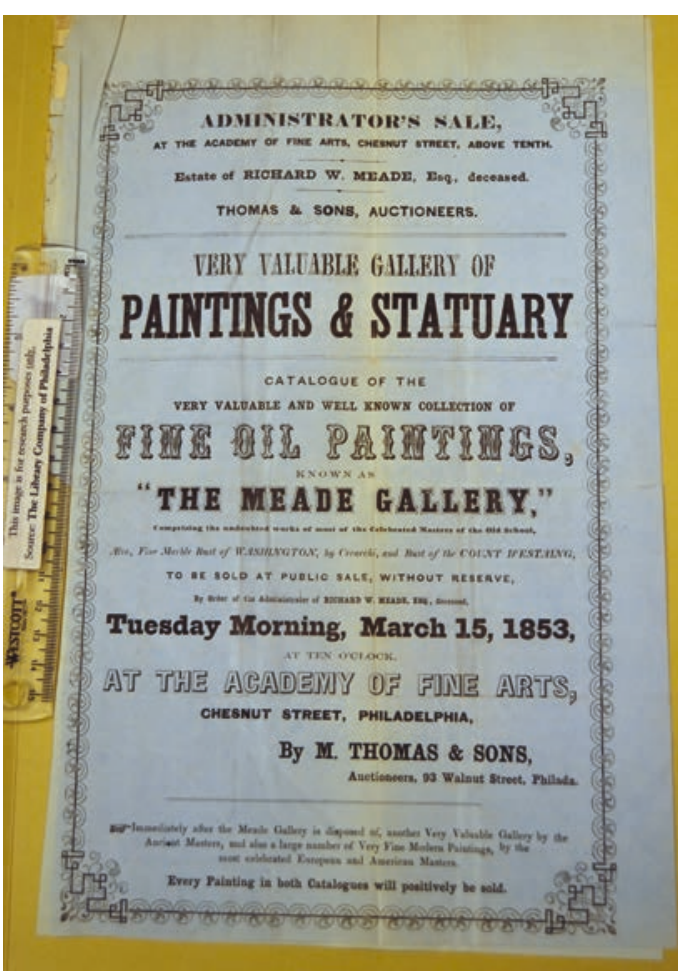

Fig 1. Portada del catálogo de la colección de arte de Richard W. Meade subastada en Filadelfia el 15 de marzo de 1853. The Library Company of Philadelphia. tellas, con su tipología y marcas; los carruajes $\mathrm{y}$ animales de tiro y finalmente figura el rebaño de 680 ovejas merinas que Meade consiguió reunir en sucesivos envíos desde Cádiz ${ }^{35}$.

La indeterminación de las fuentes respecto a los bienes inmuebles nos obliga a considerar únicamente como patrimonio de Richard W. Meade los que figuran en el inventario de 1828 y su ampliación de 1829 .

\section{La colección de pinturas de Richard W. Meade}

Como ya he señalado el inventario de los bienes de Richard W. Meade recoge varias partidas de obras de arte. Son pinturas y esculturas que constituyen una de las primeras colecciones de arte europeo llegadas a Estados Unidos a principios del siglo XIX ${ }^{36}$ y que por tanto pudieron servir para modelar el gusto de la sociedad y de los artistas estadounidenses, al disponer de las obras de los más celebrados artistas europeos para inspirarse $\mathrm{e}^{37}$. La colección según el inventario estaba formada por 136 oil paintings of different kinds and sizes, 7 family portraits, 10 family miniatures, one marble bust of Washington, one marble bust of count d'Estagne, todo ello valorado en 1.205 dólares, de los cuales 1.000 dólares correspondían a las 136 pinturas y 100 al busto de Washington. El inventario no ofrece ninguna información adicional sobre las pinturas. Sin embargo, las exposiciones que se hicieron posteriormente permiten ir configurando el grueso de la colección y perfilando el interés progresivo de la familia por la venta de una parte de las obras. En mayo de 1828, antes de su muerte, Richard Meade prestó al Athenaeum de Boston para la exposición de Old Masters, la pintura reseñada como"An early picture the late B. West" ${ }^{38}$. Dos años después en el mismo Athenaeun junto a obras de otras colecciones su viuda prestó nueve pinturas para ser vendidas (Cuadro 1).

En 1831 reaparece la colección de Meade cuando el New York Mirror anuncia ${ }^{39}$ la celebración de una exposición pública en la National Academy of Design de la colección de pinturas de Richard W. Meade e informando que se había publicado un catálogo de la serie de pinturas colected in Europe, and brought to this country by Richard W. Meade, Esq.

Ante esta noticia la primera pregunta que surge es dónde pudo reunir R.W. Meade esta colección y, si como parece probable fue en Cádiz, entonces la cuestión sería averiguar en qué momento de su estancia en España adquirió las obras y cuándo y cómo llegaron a Estados Unidos.

35 HSP, Cadwalader Papers, Legal Papers, Box 13

${ }^{36}$ Se sabe que Thomas Jefferson tenía una hermosa colección de pintura europea, pero quizás las colecciones más citadas en estas primeras décadas del siglo XIX fueron las de José Bonaparte, exiliado en New Jersey hasta 1841 y la Richard W. Meade.

${ }^{37}$ Howat/ Voorsanger, 2000: 86

38 The fourth exhibition in the Gallery of the Boston Athenaeum. 1830. Boston.

39 "Exhibition of Paintings, Colected in Spain by the Late Richard W. Meae, Esq.", New York Mirror, 17 de septiembre de 1831, pp. 86-87. 
Cuadro 1: Pinturas expuestas en el Boston Athenaeun en 1830 propiedad de Mrs. Meade

\begin{tabular}{|c|l|l|}
\hline Numero & \multicolumn{1}{|c|}{ Título } & \multicolumn{1}{c|}{ Autor } \\
\hline 11 & Vision of St. Anthony & Luca Giordano \\
\hline 15 & Landscape with cattle & Salvador Rosa \\
\hline 19 & Martyrdom of St. Lawrence & Titian \\
\hline 26 & Landscape with cattle & Salvador Rosa \\
\hline 30 & St. Francis doing Penance & Unknown. Ascribed to Tintoretto \\
\hline 67 & St. Peter weeping & Llorente \\
\hline 68 & St. Anthony with the Infant & Unknown \\
\hline 75 & Jacob wrestling with the Angel & Domenichino \\
\hline 28 & Joseph and his Brethren & Unknown \\
\hline
\end{tabular}

Fuente: The North American Review, vol. 31, 69 (Oct., 1830) pp. 309-337.

En 1810, el temor a que las tropas francesas entraran en Cádiz o que el asedio de la ciudad se prolongase, aconsejó a Meade y a otros estadounidenses repatriar a sus familias ${ }^{40}$. Seguramente su primera intención fue trasladarse con ellos a Estados Unidos, aunque algo más tarde, una vez que hubiera dejado sus negocios a cargo de personas de confianza. Esta es la impresión que proporciona el análisis del contenido del manifiesto de carga del barco de bandera estadounidense Romp, que llegó a Filadelfia procedente de Cádiz el 20 de agosto ${ }^{41}$. A bordo viajaba junto a la familia de Meade, buena parte del mobiliario de su casa y su biblioteca de la que se registran con detalle los volúmenes y sus títulos ${ }^{42}$. Pero finalmente Richard Meade, no regresó a Filadelfia, sino que permaneció en Cádiz durante toda la guerra pendiente de los préstamos que había hecho a la Regencia. La amortización de estos no se realizaría en el plazo acordado, por lo que la relación contractual con el gobierno español se complicó. El nerviosismo de Meade por la falta de liquidez a la que le habían conducido estas operaciones financieras, le llevó a publicar ciertos panfletos ofensivos que fueron la causa de su cautiverio en el Castillo de Santa Catalina durante 1812. Así pues, la única manera de recuperar sus préstamos era estar cerca de sus deudores y por tanto continuar en España. La familia regresaría a finales de 1812 y parece que permaneció en Cádiz hasta 1816. En noviembre de ese año, la mujer y los hijos de Meade volverían a embarcar hacia Filadelfia a bordo del barco estadounidense Strong junto al resto de los muebles y varias cajas con pinturas, según explica el manifiesto de carga. Lamentablemente en este documento no se dan detalles acerca del número y del contenido de las cajas que salieron de Cádiz con destino a Filadelfia.

La decisión de enviar a la familia de nuevo a Filadelfia con el resto de sus propiedades en España, respondía ahora a la complicación de su situación personal y financiera. En mayo de 1816, Meade volvió a prisión como consecuencia de ciertos negocios ilegales o irregulares en los que se vio envuelto a causa de los apuros económicos por los que atravesaba. Dos años después las autoridades le liberaron de su confinamiento, gracias a las presiones del gobierno de Estados Unidos en favor de su compatriota. Por tanto, es admisible pensar que ante sus difíciles circunstancias en España, Meade pensara que lo más sensato sería poner a salvo el resto de sus propiedades y sobre todo su colección de obras de arte, que por otro lado representaba un seguro económico a su regreso a Estados Unidos, después de sus fallidos negocios en España. Su salida

40 National Archives and Record Administration (NARA), RG 36 Records of The Bureau Of Customs. Inward Foreign Manifests, Aug1, 1810-Aug 31,1810. Box 119 NC-154 Entry 1059.

${ }^{41}$ NARA, RG 36 Records of The Bureau of Customs. Inward Foreign Manifests, Aug1, 1810-Aug 31,1810. Box 119 NC-154 Entry 1059.

${ }^{42}$ Los muebles y los 211 libros aparecen consignados a Margaret Meade, su esposa, que al mismo tiempo figura como pasajera. NARA, RG 36 Records of The Bureau of Customs. Inward Foreign Manifests, Aug1, 1810-Aug 31,1810. Box 119 NC-154 Entry 1059. 
definitiva de España tuvo lugar en 1820, cuando embarcó en Cádiz con destino a Nueva York. Es posible también, que si no había repatriado todas sus posesiones en España en 1816, aprovechara este viaje definitivo para llevar consigo el resto de sus bienes. Este extremo no se ha podido verificar, ya que solo se han consultado en los archivos federales de Estados Unidos las entradas y salidas del puerto de Filadelfia.

Si en 1810 la familia no repatrió junto a los muebles y libros, las pinturas y, sin embargo, sí se enviaron a Filadelfia en 1816, pudo ser porque la mayor parte de las pinturas de su colección se pudieron reunir durante la invasión francesa de la península y que por tanto Meade se pudo beneficiar de su condición de comerciante neutral en la compra de pinturas incautadas por los franceses.

La muerte sorprendió a Meade en Washington, en 1828, cuando reclamaba el dinero que, según el tratado firmado por Estados Unidos con España, el gobierno estadounidense debía pagarle. Los 378.879,88 dólares suponían una suma considerable que hubiera podido resolver la maltrecha situación financiera de Meade y su familia. Pero el gobierno estadounidense se negó a pagar, y la familia Meade buscó otras vías para poder sufragar la educación de los hijos menores de edad. La venta de la colección artística representaba una salida sencilla a los problemas económicos. Tres años después de la muerte de Meade el New York Mirror anunciaba ${ }^{43}$ la celebración de una exposición pública en la National Academy of Design de la colección de pinturas de Richard W. Meade que se recogían en el catálogo que esta institución publicó con el título de Catalogue of Italian, Spanish, flemish and dutch paintings, collected in Europe, and brought to this country by Richard W. Meade, Esq ${ }^{44}$. No se trata de una subasta, sino de una exposición donde era posible adquirir las pinturas o esculturas exhibidas, según se explicaba en el catálogo, a los mismos precios que Meade pagó por ellas. La familia proporcionó a la National Academy of Design solo 87 pinturas y esculturas (véase apéndice). Cada pieza aparece con su título, dimensiones, precio, autor cuando se conoce, algunos detalles técnicos o de su estado de conservación ${ }^{45}$, una sucinta explicación sobre el autor o la corriente artística a la que se adscribe la obra y una descripción de ella, mas minuciosa cuando se trata de escenas religiosas.

En 1853, después de esta primera exposición y a causa de la muerte de su esposa Margaret Coates Meade, la familia decide poner nuevamente en venta la colección, organizando una subasta en la Academy of Fine Arts de Filadelfia. El catalogo que se publicó al efecto contenía 103 obras de arte pertenecientes a la Meade Gallery ${ }^{46}$ (véase apéndice) con escasa información sobre cada una de las piezas (título, autor y dimensiones) ya que los organizadores de la subasta consideraban que puesto que la colección había sido exhibida años atrás no era necesario in preparing the catalogue to expatiate upon their merits. Tampoco se incluyó nada acerca de los precios tal vez porque según se explica en la guía de la subasta, existió un segundo catálogo que se publicó diez días antes de la venta pero que lamentablemente no se ha podido localizar y que pudo contener los precios de salida y algunos detalles más.

Dado que en ninguno de los dos catálogos se expone la totalidad de las obras, el cotejo de ambos documentos ha permitido una aproximación bastante exacta al contenido de la colección. Entre ambos documentos figuran un total de 129 piezas distintas, de las 136 obras que figuran en el inventario, y entre las que no se cuentan los retratos y miniaturas familiares. La práctica totalidad de las obras que forman la colección son pinturas, a excepción de dos bustos de mármol uno de Washington realizado por Giuseppe Ceracchi y otro representando al conde de Estaing de Jean Antoine Houdon. De las 127 pinturas descritas entre los dos catálogos, 65 se atribuyen a autores determinados sin ningún género de duda, otros 7 cuadros se asignan a escuelas pictóricas concretas, como la escuela italiana o la escuela Rubens o la escuela de Murillo, en el caso de dos

\footnotetext{
43 "Exhibition of Paintings, Colected in Spain by the Late Richard W. Meae, Esq.", New York Mirror, 17 de septiembre de 1831, pp. 86-87.

${ }_{44}$ Frick Art Reference Library, NY, The Frick Collection, ND454. M33, 1831.

45 Por ejemplo el retrato de Nicolas Antonio atribuido a Alonso Cano explica que "this picture is somewhat injured”. Frick Art Reference Library, NY, The Frick Collection, ND454. M33, 1831.

46 Catalogue of the very valuable and well known collection of fine oil pintings, known as "The Meade Gallery" [...] in The Academie of Fine Arts, March 15, 1853.
} 
cuadros los catálogos difieren en la originalidad de las obras (Murillo o copia de Murillo) y las 55 pinturas restantes no se atribuyen a ningún autor o escuela pictórica.

Predominan las obras de temas profanos, 63 son paisajes y naturalezas muertas, entre los que hay algunas marinas y escenas de género, 45 son pinturas religiosas, seis son obras que representan temas mitológicos y hay diez retratos de distintos personajes (Washington, un juez, un colegial, un anciano, el general Ballesteros...).

Entre los autores reconocidos que están presentes en la colección con varias obras, figuran Velázquez, Van Dick, Rubens, Lucas Jordán, Murillo, Tintoretto, Carreño, Sánchez Cotán, Guido Abattini, Bassano, Mario Nuzzi, Tiziano, Ribera. De otros como Alonso Cano, Caravaggio, Goya, El Greco, Gilbert Stuart, Rafael Mengs, Rembrandt o Veronés solo aparece una obra. Las atribuciones que aparecen en estos catálogos generalmente se basan en un conocimiento limitado del arte español y una preocupación por fomentar el mercado de obras de arte en crecimiento, por eso no figuran en ninguno de los catálogos referencias a la verificación de la autenticidad de las obras. Únicamente en el catálogo de 1853 se avisa al público de que every painting is sincerely believed to be an undoubted original by the artista named dejando entrever que en el caso de que alguna de las obras fuera una falsificación o la autoría fuera errónea, la casa de subastas quedaba liberada de responsabilidad alguna al no haber intencionalidad en el error. Las dificultades a la hora de establecer la autoría de algunas pinturas quedan patentes en las disparidades que surgen entre ambos catálogos. Por ejemplo en 1831 una pintura pequeña en cobre titulada St. Anthony and the Infant se atribuye a Murillo mientras que en el catálogo de 1853 aparece St. Anthony, with Infant in his arms de Luca Giordano también en cobre y del mismo tamaño. Inicialmente parecen ser la misma pintura aunque es arriesgado afirmarlo sin contar con las descripciones detalladas en ambos cuadros. Otro caso peculiar aparece en el catálogo de 1831 acerca del cuadro titulado El martirio de San Lorenzo, atribuido a Tiziano, donde se describe con detalle dicha pintura, afirmando que fue adquirida por Meade a los franceses que previamente la habían robado en Madrid. Sin embargo, la pintura no salió nunca de España y se ha conservado en su emplazamiento original hasta hoy, por tanto lo que Meade compró fue una copia.

En el catálogo de 1853 aparecían dos retratos atribuidos a Velázquez que no habían sido expuestos previamente en 1831. Parece que uno de esos retratos pudo ser el de la reina Mariana que actualmente se conserva en la New-York Historical Society, cuyo autor, en realidad es Juan Bautista Martínez del Mazo, y que fue donada a esta institución en 1867 por Thomas Jefferson Bryan quien lo había adquirido como Portrait of Infanta Margarita de Velázquez a la colección de Richard W. Meade según señala Jose Luis Colomer ${ }^{47}$. En realidad en la subasta de la Academy of Fine Arts de Filadelfia se expusieron dos retratos atribuidos a Velázquez, Portrait of the Lady who was betrothed to the son of Philip II of Spain (The Pearls and Dress are said to be inimitable) y Portrait of Maria Theresa, daughter of Philip II. Naturalmente hay que considerar que hay un error en el ordinal del rey pues debía referirse en los dos casos a Felipe III. Si efectivamente Thomas Jefferson Bryan adquirió en la subasta de 1853 el retrato que se expone en la New-York Historical Society, hemos de aceptar que en realidad compró el Portrait of the Lady who was betrothed to the son of Philip II of Spain, aunque la anotación acerca de las características perlas debe referirse al Portrait of Maria Theresa, daughter of Philip II que se conserva en el Metropolitan Museum de Nueva York y atribuido a la escuela de Velázquez.

Como he dicho anteriormente, antes de 1810 no tenemos noticias de que Richard Meade hubiera adquirido ninguna de las obras que se reseñan en los catálogos de 1831 y 1853 . Tampoco los documentos desvelan el origen de la colección, sólo un apunte en una de las obras del catálogo de 1831 nos da la clave de cómo pudo reunirse una colección tan centrada en la pintura española e italiana de los siglos XVI y XVII. En dicho catalogo aparece reseñada la pintura de El Martirio de San Lorenzo atribuido a Tiziano, con algunos datos biográficos de Tiziano y explicando la génesis del cuadro, que fue encargada por Felipe II y se añade was removed by the french army and purchased by Mr. Meade in the neighbourhood of Cadiz, por 5.000 dólares $^{48}$. Si Meade compró a los

47 Reist/ Colomer, 2012: 254.

48 Frick Art Reference Library, NY, The Frick Collection, ND454. M33, 1831. 
franceses esta pintura, es muy probable que la mayoría de las obras de su colección fueran adquiridas del mismo modo, aprovechando el expolio que los franceses realizaron en iglesias y palacios españoles y beneficiándose probablemente de la condición de ciudadano de un estado neutral para poder negociar con ellos sin demasiados riesgos. No obstante, tampoco hay que descartar la hipótesis, de que algunas obras las consiguiera Meade en pago a deudas pendientes, esta es al menos la razón que aduce alguno de los biógrafos de su hijo el general George Gordon Meade ${ }^{49}$; sin embargo sin la contabilidad privada de su casa comercial es muy difícil constatar este extremo.

Meade también pudo adquirir algunas de las piezas en Madrid en los diversos viajes que hizo a la capital. De hecho debió encargar al pintor valenciano Vicente López su retrato en una de esas visitas en el año 1815. Y es probable que también comprara allí o al menos contactara con algún marchante de arte para comprar el busto de Washington que la viuda de José Jáudenes le vendió por 2.000 dólares $^{50}$ después de 1812 , fecha en la que enviudó.

He intentado averiguar si algunas de las pinturas de la colección de Meade pudieron ser compradas en Cádiz. De hecho en las mismas fechas en que Meade residió en la ciudad se formalizó la partición de los bienes de Sebastián Martínez, un rico comerciante riojano mencionado más arriba. Los gastos elevados que se derivaban de una liquidación de bienes testamentarios podía ser una oportunidad para conseguir obras de arte a buen precio. Al respecto el conde Maule asegura en sus memorias que una de las hijas y herederas de Sebastián Martínez vendió las obras de arte que le correspondieron a los ingleses. Pudo suceder que en esas circunstancias alguno de los cuadros fuera adquirido por Meade. Sin embargo, la descripción del documento notarial donde figuran inventariados los cuadros de la "hijuela" de Catalina ${ }^{51}$ en la partición de Sebastián Martínez, es tan esquemática, que inferir en el caso de algunas pinturas solo con la semejanza de los títulos que Meade la compró es simplemente una especulación.

Las 136 oil painting del inventario de 1828 fueron valoradas en 1.000 dólares por los tasadores de la Orphan's Court de Washington ${ }^{52}$. Tres años después en el catálogo de la exposición de 1831 en 82 de las 87 obras recogidas se expresaban los precios a los que se dice que Meade compró cada una de las pinturas. En la correspondencia que su hija Margaret mantuvo en 1845 con el abogado encargado de la administración de los bienes de su padre y del cumplimiento de su testamento, esta criticaba a los tasadores que infravaloraron las propiedades de su padre y en concreto de our valuable colection of paintings, pues consideraba que había algunos cuadros que podían valer alrededor de 5.000 dólares y que en conjunto la colección podía superar los 50.000 dólares ${ }^{53}$. Esta apreciación no estaba demasiado alejada de la realidad ya que el importe total de las obras del catálogo de 1831 ascedió a 38.154 dólares. Margaret también se quejaba en sus cartas de que su madre fuera la única custodia de la colección y que solo ella podía decidir sobre su distribución, con el riesgo de favorecer a algunos miembros de la familia, a pesar de que su padre no hizo diferencias entre sus hijos. Pero lo más interesante de esta correspondencia es que Margaret Meade, revela cuál fue el motivo por el que su padre invirtió en la compra de las pinturas. Según explica su hija, Meade quería dejar a sus hijos una colección de arte y al mismo tiempo una fortuna que les permitiría vivir sin partir de cero, lo que lleva a pensar que consideraba el arte como una inversión muy provechosa a medio y largo plazo ${ }^{54}$.

La existencia de los dos catálogos responde claramente a las necesidades económicas por las que pasó la familia Meade en dos momentos críticos, primero después de la muerte de Richard W. Meade (1828) el de 1831, cuando la mayoría de los hijos eran todavía menores de edad; y luego, tras la muerte de la esposa de Meade (1852) el de 1853, cuando todavía algunos de los

49 Pennsylvania Magazine of History and Biography, vol. XXIV (1900), p. 243.

${ }^{50}$ Catalogue of the very valuable, p. 4.

51 Archivo Histórico Provincial de Cádiz (AHPC), Protocolo 5387, ff. 1235-1317.

${ }^{52}$ HSP, Cadwalader Papers, Legal Papers, Box 13.

53 Carta de Margaret Meade, hija, a John Cadwalader, Washington City 2 de noviembre de 1843. HSP, Cadwalader Papers. Legal Papers. Box 13.

${ }^{54}$ Carta de Margaret Meade, hija a John Cadwalader, Washington City 8 de octubre de 1843 . HSP, Cadwalader Papers. Legal Papers. Box 13. 
miembros de la familia tenían dificultades económicas y había que repartir los bienes que aún quedaban por distribuir y especialmente la colección de obras de arte. En ambos casos, está claro que la decisión pasó por la venta de las pinturas y esculturas.

Para la exposición y venta del año 1831 se eligieron algunas obras valiosas como El Martirio de San Lorenzo atribuida a Ticiano, cuyo precio de compra y venta estaba en 5.000 dólares y $L a$ llamada de San Mateo de Lucas Jordán, que aparecía con un precio de 7.000 dólares, pero sobre todo se optó por piezas más asequibles económicamente para los compradores cuyos precios oscilaban entre los 100 y los 1000 dólares, así como un número considerable de cuadros de pequeño formato, la mayoría paisajes y marinas que salieron a la venta por precios más económicos, entre los 10 y los 80 dólares. Dado que un buen número de las pinturas de este último grupo no aparecen en el catálogo de la subasta de 1853, es razonable pensar que se vendieron en la exposición de 1831. En esta primera exposición no fueron adquiridas las obras de mayor formato y más caras de tema mayoritariamente piadoso ${ }^{55}$, lo que podría indicarnos que por estos años en Nueva York el mercado de obras de arte no era propicio todavía ni para las pinturas de tema religioso ni para la pintura barroca española, ni siquiera a modo de inversión. El gusto de las clases pudientes protestantes de Nueva York era más amigo de la pintura flamenca y holandesa y de temas costumbristas y paisajes, con tamaños medianos y pequeños, más acorde con el tipo de viviendas en que residían. Todavía tardaría en calar en el ánimo de los estadounidenses el gusto por lo español que propagó Washington Irving en sus $\operatorname{libros}^{56}$ y que no se instalaría entre algunos coleccionistas estadounidenses hasta finales del siglo XIX. No obstante, llama la atención el hecho de que ni siquiera se vendió el retrato de Washington, uno de los que pintó Gilbert Stuart y cuyo precio era de 100 dólares o el busto de mármol también de Washington que estaba valorado en 2.000 dólares.

La subasta celebrada en Filadelfia en 1853 vuelve a poner de manifiesto el interés de la familia por convertir en dinero el legado de Meade. En este caso el público era ciertamente diferente al existir en Filadelfia una población católica numerosa y por tanto más proclive al gusto por los temas de carácter religioso. También hay que tener en cuenta los veinte años transcurridos desde la exposición de Nueva York que añadían al mercado de arte estadounidense un mayor conocimiento de la pintura barroca española y europea. La subasta se celebró en la Academia de Bellas Artes de Filadelfia siete años después de que un incendio destruyera parte de sus fondos, poniéndose a la venta la práctica totalidad de la colección, salvo los retratos familiares.

No ha sido posible encontrar documentos que atestigüen quienes fueron los compradores en la subasta de 1853. Parece que no todo se vendió y que hubo algunas obras que pudieron quedar en poder de la familia, bien porque se les adjudicara en un reparto previo a la subasta o porque su elevado precio impidió que encontraran un comprador. Este pudo ser el caso del cuadro titulado La llamada de san Mateo de Lucas Jordán, pues su presencia en los dos catálogos muestra el interés de la familia por venderlo, sin embargo, parece que no hubo compradores interesados, tal vez por su elevado precio, por su tamaño, tal vez por el tema o tal vez por su estilo.

Las obras que no se vendieron en 1853 parece que se distribuyeron entre los herederos y estos posteriormente fueron vendiéndolas a particulares o instituciones. El cuadro de Lucas Jordán anteriormente mencionado fue vendido por Martha Meade a la Universidad de Georgetown en 1860 y la esposa de Robert, uno de los hijos de Meade, vendió el busto de Washington al Governeur Kemble y actualmente se conserva en el Metropolitan Museum de Nueva York.

La familia Meade no incluyó ninguno de los retratos familiares en los catálogos. Sabemos de la existencia de un retrato de Richard W. Meade y otro de su esposa pintados por Gilbert Stuart y que según reza en una nota el catálogo de 1858, has never been out of the possession of the family. Además existe un retrato del propio Richard W. Meade que se conserva en el Meadows Museum de Dallas realizado en 1815 en Madrid por el pintor valenciano Vicente López ${ }^{57}$.

55 No se vendió ni El Martirio de San Lorenzo de Ticiano ni La llamada de San Mateo de Lucas Jordán.

56 Kagan, 2010: 37-58.

${ }^{57}$ La pasión por España de Algur H. Meadows se reflejaba en su idea de crear un "pequeño Prado en Texas". The Meadows Museum es un monumento a su capacidad para comprar y exhibir pinturas españolas. 


\section{Conclusiones}

Fueron muy pocos los comerciantes estadounidenses que aprovechando las buenas previsiones comerciales que les otorgaba el periodo de guerras a finales del siglo XVIII y primeros años del XIX se trasladaron a España. Richard W. Meade fue el más conocido, no solo por sus negocios sino por su implicación económica y política con el gobierno de la Regencia. Aprovechando su estancia en Cádiz durante los años de la invasión francesa de la península, logró reunir una importante colección de obras de arte, recurriendo en algunos casos a la compra de obras expoliadas por los franceses. En 1816, unos años antes de su regreso a Filadelfia, sacó las pinturas de Cádiz en el barco estadounidense Strong. La colección, una de las primeras de arte barroco llegadas a Estados Unidos, fue adquirida como una inversión que la familia utilizó para enderezar su maltrecha situación económica, ofreciéndola al público de Nueva York, primero, y Filadelfia, después, en dos subastas, en 1831 y 1851. Las pinturas de temas religiosos y gran formato no fueron del gusto del público estadounidense que prefería las obras más pequeñas con temas de paisajes y escenas de género, más adecuadas para el tipo de viviendas en que residían. Parece que el gusto por lo español tardaría todavía unos años en arraigar en la sociedad estadounidense.

\section{APÉNDICE DOCUMENTAL}

Listado de las obras incluidas en los catálogos de 1831 y 1853.

Se presentan ambos catálogos en la misma tabla, ordenados por autores, con el año del catálogo y los precios y las dimensiones cuando aparecen.

El anuncio del catálogo de 1831 fue como sigue:

CATALOGUE OF Italian, Spanish, Flemish and Dutch PAINTINGS Collected in Europe, and brought to this Country, By RICHARD W. MEADE, Esq. now exhibiting in The Gallery of The National Academy of Design. $1831^{58}$.

El anuncio del catálogo de 1853 fue el siguiente:

ADMINISTRATOR'S SALE, AT THE ACADEMY OF FINE ARTS, CHESNUT STREET, ABOVE TENTH. Estate of RICHARD W. MEADE, Esq. deceased. Thomas \& Sons, Auctioneers. Very Valuable Gallery of Painting \& Statuary. Catalogue of the very valuable and well known collection of fine oil paintings, known as "The Meade Gallery", comprising the undoubted Works of most of the celebrated Masters of the Old School. Philadelphia, Feb., $1853^{59}$.

\begin{tabular}{|c|l|l|c|c|c|}
\hline $\begin{array}{c}\text { Núm. en } \\
\text { catálogo }\end{array}$ & \multicolumn{1}{|c|}{ Título } & Autor & $\begin{array}{c}\text { Ancho x } \\
\text { alto en } \\
\text { pulgadas }\end{array}$ & $\begin{array}{c}\text { Precio } \\
\text { \$ }\end{array}$ & Año \\
\hline 16 & Portrait of the great Nicholas Antonio & Alonso Cano & $64 x 42$ & 250 & 1831 \\
\hline 65 & Portrait of Nicholas Antonio & Bassano & $41 \times 63$ & 1853 \\
\hline 13 & The Departure from the Ark & & $69 x 50$ & 600 & 1831 \\
\hline 2 & Going out of the Ark (companion to No. 73) & & $69 \times 50$ & & 1853 \\
\hline 12 & The Entrance into the Ark & & $69 \times 50$ & 600 & 1831 \\
\hline 73 & Going into the Ark (companion to No. 2) & & $69 \times 50$ & & 1853 \\
\hline 61 & St. Peter escaping from Prison & Caravagio & & 80 & 1831 \\
\hline 100 & The Angel liberating St. Peter from prison & & $48 \times 36$ & & 1853 \\
\hline 13 & Old Books and Papers (companion to No. 20) & Cario & $34 \times 21$ & & 1853 \\
\hline 20 & Old Books and Papers (companion to No. 13) & & $34 \times 21$ & & 1853 \\
\hline 51 & Piece representing Old Books and Papers & Carreno & & 100 & 1831 \\
\hline 52 & Companion to 51 & & & 80 & 1831 \\
\hline 54 & Fowls Feeding & & & 80 & 1831 \\
\hline
\end{tabular}

58 Frick Art Reference Library ND454.M33.

59 Library Company of Philadelphia, SM \#AM 1853, Mea. 


\begin{tabular}{|c|c|c|c|c|c|}
\hline $\begin{array}{l}\text { Núm. en } \\
\text { catálogo }\end{array}$ & Título & Autor & $\begin{array}{l}\text { Ancho } x \\
\text { alto en } \\
\text { pulgadas }\end{array}$ & $\begin{array}{c}\text { Precio } \\
\$\end{array}$ & Año \\
\hline 55 & Companion to 54 & “" & & 100 & 1831 \\
\hline 58 & Domestic Fowls (companion to No. 79) & “" & $24 \times 20$ & & 1853 \\
\hline 79 & Domestic Fowls (companion to No. 58) & “" & $27 \times 20$ & & 1853 \\
\hline 86 & The bust of Washington & Ceracchi & & 2000 & 1831 \\
\hline 32 & Marble Bust of Washington & “" & & & 1853 \\
\hline 6 & Mars, Venus and Vulcan & Contrado & $39 \times 15$ & & 1853 \\
\hline 23 & Jacob Wrestling wth the Angel & Domenichino & $56 \times 47$ & 1000 & 1831 \\
\hline 7 & Jacob wrestling with the Angels & “ & $38 \times 46$ & & 1853 \\
\hline 37 & St. Peter of Alcantara & El Greco & & 250 & 1831 \\
\hline 54 & St. Peter of Alcantara & “" & $29 \times 39$ & & 1853 \\
\hline 25 & Still Life & Elder Nani & $64 \times 43$ & 200 & 1831 \\
\hline 1 & Still Life & “ & $64 \times 43$ & & 1853 \\
\hline 38 & Head of St. John the Baptist & Espagnoletto & & 200 & 1831 \\
\hline 70 & A Dutchman Smoking & Flemish School & & 30 & 1831 \\
\hline 83 & The Virgin of the Rose & Garafalo & & 12 & 1831 \\
\hline 68 & Portrait of Washington & Gilbert Stuart & $24 \times 28$ & & 1853 \\
\hline 78 & Monkeys, (oval) & Goya & $14 \times 11$ & & 1853 \\
\hline 44 & Head of the Saviour-An Oral & Guido Albatini & & 80 & 1831 \\
\hline 90 & Head of the Savior (companion to No. 91) oval & “ & $17 \times 20$ & & 1853 \\
\hline 45 & Head of the Virgin-An Oral & “" & & 80 & 1831 \\
\hline 91 & Head of the Virgin (companion to No. 90) oval & “" & $17 \times 20$ & & 1853 \\
\hline 87 & A bust in marble of the Count D'Estaing & Houdon & & & 1831 \\
\hline 33 & Marble Bust of Count D'Estaing & "“ & & & 1853 \\
\hline 26 & Landscape & “ & & 12 & 1831 \\
\hline 27 & Landscape & “ & & 25 & 1831 \\
\hline 28 & Landscape & “ & & 50 & 1831 \\
\hline 29 & Landscape & “ & & 12 & 1831 \\
\hline 36 & Madonna with Infant at the breast & “" & & 200 & 1831 \\
\hline 92 & Madonna, with Infant at the breast & "(Old) & $24 \times 29$ & & 1853 \\
\hline 57 & St. Lawrence & "(Old) & & 50 & 1831 \\
\hline 53 & St. Lawrence (companion to No. 83) & " (Old) & $24 \times 29$ & & 1853 \\
\hline 58 & St. Agnes (companion to 57) & "(Old) & & 200 & 1831 \\
\hline 63 & Still Life & “" & & 200 & 1831 \\
\hline 64 & Companion to 63 & " & & 200 & 1831 \\
\hline 75 & A Study of Hands and Feet & “ & & 50 & 1831 \\
\hline 76 & Endymion Sleeping & “" & & 50 & 1831 \\
\hline 82 & A Roman Soldier & Italian School & & 400 & 1831 \\
\hline 69 & Study of Heads & “ & $33 \times 13$ & & 1853 \\
\hline 83 & St. Ursula (companion to No. 53) & " (Old) & $24 \times 29$ & & 1853 \\
\hline 30 & Fruits and Birds & J. S. Cotan & & 200 & 1831 \\
\hline 31 & Vegetables and Fruit & “ & & 200 & 1831 \\
\hline 24 & Fruit and Vegetables (companion to No. 37) & “" & $33 \times 27$ & & 1853 \\
\hline 37 & Fruit and Vegetables (companion to No. 24) & “" & $33 \times 27$ & & 1853 \\
\hline
\end{tabular}




\begin{tabular}{|c|c|c|c|c|c|}
\hline $\begin{array}{l}\text { Núm. en } \\
\text { catálogo }\end{array}$ & Título & Autor & $\begin{array}{l}\text { Ancho x } \\
\text { alto en } \\
\text { pulgadas }\end{array}$ & $\begin{array}{l}\text { Precio } \\
\$\end{array}$ & Año \\
\hline 56 & The Three Wise men with Offerings & Juan Ant. Escalante & & 50 & 1831 \\
\hline 101 & Adoration of the Magi & “" & $48 \times 36$ & & 1853 \\
\hline 20 & St. Peter Weeping & Llorente & $58 \times 43$ & 1000 & 1831 \\
\hline 95 & St. Peter weeping & “ & $37 \times 39$ & & 1853 \\
\hline 1 & The Calling of St. Matthew & $\begin{array}{l}\text { Luca Giordano } \\
\text { (Lucas Jordan) }\end{array}$ & $117 x 93$ & 7.000 & 1831 \\
\hline 28 & Calling of St. Matthew & “ & $103 \times 81$ & & 1853 \\
\hline 50 & $\begin{array}{l}\text { Jacob and Esau Receiving their Father's } \\
\text { Blessing (copper) }\end{array}$ & “ & & 100 & 1831 \\
\hline 42 & Jacob blessings Esau, (on Copper) & “ & $13 \times 14$ & & 1853 \\
\hline 47 & Gypsy Scene, (oval) & “ & $19 \times 14$ & & 1853 \\
\hline 48 & Gypsy Scene, (oval) & “ & $19 \times 14$ & & 1853 \\
\hline 49 & Gypsy Scene, (oval) & “" & $19 \mathrm{x} 14$ & & 1853 \\
\hline 50 & Gypsy Scene, (oval) & “ & $19 \times 14$ & & 1853 \\
\hline 60 & St. Anthony, with Infant in his arms (on Copper) & “ & $8 \times 11$ & & 1853 \\
\hline 3 & The Vision of St. Anthony & “ & $102 \times 76$ & 1.200 & 1831 \\
\hline 21 & $\begin{array}{l}\text { Female with Cup and Dagger, surrounded with } \\
\text { Flowers }\end{array}$ & “ & $57 x 48$ & 400 & 1831 \\
\hline 22 & Females with Flowers & Mario Nuzzi & $57 x 48$ & 400 & 1831 \\
\hline 67 & $\begin{array}{l}\text { Female encircled by a Wreath of Flowers, } \\
\text { (companion to No. 82) }\end{array}$ & “ & $40 \times 50$ & & 1853 \\
\hline 82 & $\begin{array}{l}\text { Female encircled by a Wreath of Flowers, } \\
\text { (companion to No. 67) }\end{array}$ & “ & $40 \times 50$ & & 1853 \\
\hline 42 & St. Jerome doing penance & Mateo Zerez & & 200 & 1831 \\
\hline 72 & Vision of St. Jerome & “ & $64 \times 20$ & & 1853 \\
\hline 4 & Falls of Tivoli & Miestf & $28 \times 38$ & & 1853 \\
\hline 78 & Landscape & Miranda & & 50 & 1831 \\
\hline 79 & Landscape, with bridge and wáter scene & “ & & 50 & 1831 \\
\hline 48 & St. Anthony and The Infant & Murillo & & 100 & 1831 \\
\hline 49 & $\begin{array}{l}\text { The Martyrdom of Emeterio and Celedonio (on } \\
\text { copper) }\end{array}$ & “ & & 100 & 1831 \\
\hline 40 & $\begin{array}{l}\text { The Martyrdom of Ermiterio and Celedonio, } \\
\text { (on Copper) }\end{array}$ & “ & $9 \times 12$ & & 1853 \\
\hline 43 & The three wise men, with offerings to the Infant & “ & & 300 & 1831 \\
\hline 5 & The Adoration of the Three Kings & " & $52 \times 23$ & & 1853 \\
\hline 59 & The Visitation of Joseph and Mary to Elizabeth & “ & & 150 & 1831 \\
\hline 6 & $\begin{array}{l}\text { Vision of St. Francis receiving the saviour from } \\
\text { the cross }\end{array}$ & Murillo (Copy from) & $112 \times 74$ & 400 & 1831 \\
\hline 98 & Vision of St Francis & “ & $72 \times 108$ & & 1853 \\
\hline 9 & St. Thomas of Villanueva, giving Alms & A pupil of Murilo & $81 \times 60$ & 1.200 & 1831 \\
\hline 75 & $\begin{array}{l}\text { St. Thomas, of Villa Nueva, distributing Alms } \\
\text { to the Poor }\end{array}$ & “ & $48 \times 69$ & & 1853 \\
\hline 39 & Landscape, Valencians and Cart & Rafael Mengs & $15 \times 14$ & & 1853 \\
\hline 39 & Head of St. Peter & Ribalta & & 200 & 1831 \\
\hline 26 & Head of St. Peter & “" & $15 \times 17$ & & 1853 \\
\hline 70 & Head of John, the Baptist & Ribera & $23 \times 17$ & & 1853 \\
\hline
\end{tabular}




\begin{tabular}{|c|c|c|c|c|c|}
\hline $\begin{array}{l}\text { Núm. en } \\
\text { catálogo }\end{array}$ & Título & Autor & \begin{tabular}{|c|} 
Ancho x \\
alto en \\
pulgadas
\end{tabular} & $\begin{array}{l}\text { Precio } \\
\$\end{array}$ & Año \\
\hline 12 & Head of John, the Baptist & “ & $23 \times 16$ & & 1853 \\
\hline 88 & St. Onofrio, in penance & “" & $39 x 49$ & & 1853 \\
\hline 66 & St. Peter & “" & $36 \times 50$ & & 1853 \\
\hline 35 & Madonna with the Infant and St. John & Rubens & & 800 & 1831 \\
\hline 52 & Virgin Infant Saviour and St. John & “" & $33 \times 39$ & & 1853 \\
\hline 15 & Moses and the Brazan Serpent & “ & $62 \times 51$ & 500 & 1831 \\
\hline 34 & Moses and the Brazen Serpent & “ & $62 \times 51$ & & 1853 \\
\hline 47 & The Graces and Cupid & Rubens'School & & 100 & 1831 \\
\hline 43 & St. Michae and the Devil (on Copper) & “ & $12 \times 17$ & & 1853 \\
\hline 33 & A Landscape with troop of horse & Salvatore Rosa & & 300 & 1831 \\
\hline 57 & Landscape, with Troop of Horse & “" & $38 \times 25$ & & 1853 \\
\hline 19 & Landscape with Cattle and Waterfall & “ & $60 \times 45$ & 1000 & 1831 \\
\hline 36 & $\begin{array}{l}\text { Landscape, with Cattle and Waterfall, } \\
\text { (companion to No. 64) }\end{array}$ & “ & $53 \times 37$ & & 1853 \\
\hline 18 & Small Landscape, with Cattle & “ & $63 \times 45$ & 1000 & 1831 \\
\hline 15 & Small Landscape, with Cattle, (circular) & “ & $8 \times 8$ & & 1853 \\
\hline 16 & Small Landscape, with Cattle, (circular) & “ & $8 \mathrm{x} 8$ & & 1853 \\
\hline 18 & Small Landscape, with Cattle, (circular) & “ & $8 \times 8$ & & 1853 \\
\hline 19 & Small Landscape, with Cattle, (circular) & “" & $8 \mathrm{x} 8$ & & 1853 \\
\hline 22 & Small Lanscape, (circular) & “ & $8 \times 8$ & & 1853 \\
\hline 23 & Small Landscape with Cattle, (circular) & “ & $8 \mathrm{x} 8$ & & 1853 \\
\hline 64 & Landscape, with Cattle, (companion to No 36) & “ & $53 \times 37$ & & 1853 \\
\hline 71 & Battle Piece, between the Spaniards and Saracens & “" & $39 \times 25$ & & 1853 \\
\hline 77 & Portrait of Washington & Stewart & & 100 & 1831 \\
\hline 32 & A Dark Landscape & Swanewelt & & 100 & 1831 \\
\hline 11 & Landscape representing Evening & Tempesta & $67 x 43$ & 1.250 & 1831 \\
\hline 10 & Landscape representing Morning & “ & $67 \times 43$ & 1.250 & 1831 \\
\hline 3 & Landscape, Evening Scene, (do. to No. 29) & “" & $67 \times 48$ & & 1853 \\
\hline 29 & Landscape, Morning Scene, (companion to No. 3) & “ & $67 \mathrm{x} 48$ & & 1853 \\
\hline 8 & Flemish Feastival (companion to no. 25) & Teniers & $53 \times 39$ & & 1853 \\
\hline 25 & Flemish Festival, (companion to No. 8) & Teniers & $53 \times 39$ & & 1853 \\
\hline 80 & Dark Landscape & Tintoretto & $23 \times 14$ & & 1853 \\
\hline 81 & Dark Landscape & " & $23 \times 14$ & & 1853 \\
\hline 2 & The Martyrdom of St, Lawrence & Titian & $91 \times 77$ & 5.000 & 1831 \\
\hline 74 & Martyrdom of St. Lawrence & “ & $78 \times 63$ & & 1853 \\
\hline 93 & Portrait of a Roman Lady & “" & $39 x 43$ & & 1853 \\
\hline 34 & A Magdalen & Vandyck & & 1.000 & 1831 \\
\hline 76 & Mary Magdalene & “ & $31 \times 51$ & & 1853 \\
\hline 53 & Portrait of a Judge & “ & & 50 & 1831 \\
\hline 96 & Portrait of a Judge & “" & $22 \times 39$ & & 1853 \\
\hline 24 & A Piece of still Life & Velazquez & $21 \times 27$ & 400 & 1831 \\
\hline 51 & Two Brothers of Joseph & “" & $32 \times 31$ & & 1853 \\
\hline 55 & $\begin{array}{l}\text { Portrait of the Lady who was betrothed to the } \\
\text { son of Phillip II of Spain.(The Pearls and Dress } \\
\text { are said to be inimitable) }\end{array}$ & “ & $23 \times 28$ & & 1853 \\
\hline
\end{tabular}




\begin{tabular}{|c|c|c|c|c|c|}
\hline $\begin{array}{l}\text { Núm. en } \\
\text { catálogo }\end{array}$ & Título & Autor & \begin{tabular}{|c} 
Ancho $x$ \\
alto en \\
pulgadas
\end{tabular} & $\begin{array}{c}\text { Precio } \\
\$\end{array}$ & Año \\
\hline 56 & Portrait of Maria Theresa, daughter of Phillip II & “ & $24 \times 27$ & & 1853 \\
\hline 77 & Head of a Friar & " & $18 \times 24$ & & 1853 \\
\hline 85 & Still Life & Velazquez & $75 \times 27$ & & 1853 \\
\hline 5 & $\begin{array}{l}\text { The Saviour expounding the law to the doctors } \\
\text { inthe temple }\end{array}$ & Veronese & $157 \times 75$ & 1.200 & 1831 \\
\hline 85 & Flower Pieces & Zarbara & & 12 & 1831 \\
\hline 4 & St. Francis doing Penance & & & 12 & 1831 \\
\hline 7 & The Prodigal Sons's Return & & $75 \times 68$ & 25 & 1831 \\
\hline 8 & The Brethren of Joseph with Presents & & $87 x 68$ & 25 & 1831 \\
\hline 14 & St. Joseph with the infant & & $65 \times 47$ & 30 & 1831 \\
\hline 17 & St. Anthony with the Infant & & $57 \times 42$ & 30 & 1831 \\
\hline 40 & Head of a Young Collegian & & & 30 & 1831 \\
\hline 41 & Head of an Old Man & & & 30 & 1831 \\
\hline 46 & St. Michael and the Devil (on Copper) & & & 30 & 1831 \\
\hline 60 & The day of Judgment & & & 30 & 1831 \\
\hline 62 & Subject not Known & & & 200 & 1831 \\
\hline 65 & Dutch Naval Pieces & & & 200 & 1831 \\
\hline 66 & Dutch Naval Pieces & & & 10 & 1831 \\
\hline 67 & Dutch Naval Pieces & & & 12 & 1831 \\
\hline 68 & Dutch Naval Pieces & & & 150 & 1831 \\
\hline 69 & A Bird with Sweetmeats & & & 1.200 & 1831 \\
\hline 71 & Companion to 70 & & & 200 & 1831 \\
\hline 72 & Two Dutchmen Eating & & & 100 & 1831 \\
\hline 73 & Companion to 72 & & & 25 & 1831 \\
\hline 74 & Likeness of Gen. Ballesteros & & & 500 & 1831 \\
\hline 80 & A Small Landscape & & & 100 & 1831 \\
\hline 81 & A Small Landscape & & & 500 & 1831 \\
\hline 84 & Monkey Eating Fruit & & & 30 & 1831 \\
\hline 88 & Two elegant Vases & & & & 1831 \\
\hline 9 & A Head & & $12 \times 17$ & & 1853 \\
\hline 10 & $\begin{array}{l}\text { Italian Garden and Villa (companion to Nos. } \\
11,17 \text { and } 21 \text { ) }\end{array}$ & & $32 \times 25$ & & 1853 \\
\hline 11 & $\begin{array}{l}\text { Italian Landscape, with Falls, \& c. (companion } \\
\text { to Nos. } 10,17 \text { and 17) }\end{array}$ & & $32 \times 25$ & & 1853 \\
\hline 14 & Diana and Nymphs (on Copper) & & $21 \times 16$ & & 1853 \\
\hline 17 & $\begin{array}{l}\text { Italian Garden and Villa, (companion to } \\
\text { Nos.10,11 and 21) }\end{array}$ & & $32 \times 25$ & & 1853 \\
\hline 21 & $\begin{array}{l}\text { Italian Landscape, with Villa, (companion to } \\
\text { Nos, 10,11 and 27) }\end{array}$ & & $32 \times 25$ & & 1853 \\
\hline 27 & Joseph and Infant Saviour & & $46 \times 64$ & & 1853 \\
\hline 30 & Two Pieces, Dutchmen Smoking & & $6 \times 8$ & & 1853 \\
\hline 31 & Two Pieces, Dutchmen Eating and Drinking & & $9 \mathrm{x} 6$ & & 1853 \\
\hline 35 & St. Anthony, with Infant in his arms; a Vision & & $42 \times 56$ & & 1853 \\
\hline 38 & Landscape, Road-side figures & & $14 \times 9$ & & 1853 \\
\hline 41 & Sparrow, with Sweetmeats & & $12 \times 9$ & & 1853 \\
\hline
\end{tabular}




\begin{tabular}{|c|l|c|c|c|c|}
\hline $\begin{array}{c}\text { Núm. en } \\
\text { catálogo }\end{array}$ & & Autor & $\begin{array}{c}\text { Ancho x } \\
\text { alto en } \\
\text { pulgadas }\end{array}$ & $\begin{array}{c}\text { Precio } \\
\mathbf{S}\end{array}$ & Año \\
\hline 44 & Kettles \& c. & & $10 \times 8$ & & 1853 \\
\hline 45 & Bread and Wine & & $10 \times 8$ & & 1853 \\
\hline 46 & Two Small Marine Pieces (companions to No 59) & & $9 \times 7$ & & 1853 \\
\hline 59 & Two Small Marine Pieces (companions to No. 46) & & $9 \times 7$ & & 1853 \\
\hline 61 & Small Figure & & $9 \times 12$ & & 1853 \\
\hline 62 & Portrait of General Ballesteros & & $18 \times 25$ & & 1853 \\
\hline 63 & Three wáter Colored Pintings of Iturbide's & & $28 \times 19$ & & 1853 \\
\hline 84 & Coronation & Joseph's Brethren with presents & & & \\
\hline 86 & Two Old Flowers Pieces & & & & 1853 \\
\hline 87 & Two Oil colored Prints & & $21 \times 26$ & & 1853 \\
\hline 89 & Portrait & & $87 \times 68$ & & 1853 \\
\hline 94 & Return of the Prodigal Son & & $12 \times 8$ & & 1853 \\
\hline 97 & Endymion sleeping & & $48 \times 36$ & & 1853 \\
\hline 99 & Communion of St. Theresa & & $48 \times 36$ & & 1853 \\
\hline 102 & Day of Judgment & $48 \times 36$ & & 1853 \\
\hline 103 & Virgin of the Rose & & \\
\hline
\end{tabular}

\section{BIBLIOGRAFÍA}

Antigüedad del Castillo-Olivares, M. Dolores/Alzaga Ruiz, Amaya (eds.) (2011): Coleccionismo, expolio, museos y mercado artístico en España en los siglos XVIII y XIX. Madrid: Editorial Universitaria Ramón Areces.

Antón Solé, Pablo (1966): “El anticuario gaditano Pedro Alonso de O’Crowley”. En: Archivo Hispalense, 136, Sevilla, pp. 151-165.

Bustos Rodríguez, Manuel (2005): Cádiz en el sistema Atlántico. La ciudad, sus comerciantes y la actividad mercantil (1650-1830), Cádiz: Sílex.

Carrasco-González, Guadalupe (2009): “El comercio y la gestión de los negocios estadounidenses en Cádiz a través de su consulado (1785-1807)”. En: Dubet, Isidro (ed.): El mar en los siglos modernos, Santiago de Compostela: Xunta de Galicia, pp. 305-318.

Carrasco-González, Guadalupe (2010): "La délégation consulaire des État-Unis à Cadix au debut du XIXe sècle". En: Ulbert, Jörg/Prijac, Lukian (eds.): Consuls et services consulaires au XIX siêcle, Hamburg: DOBU Verlag, pp. 234-247.

Carrasco-González, Guadalupe (2015): "Richard Meade: Negocios y desventuras de un estadounidense en Cádiz (18041820)". En: Iglesias, Juan José (ed.): Comercio y Cultura en la Edad Moderna. Sevilla: Universidad.

Carrasco-González, Guadalupe (2016): "El comercio de Estados Unidos con Cádiz entre 1788 y 1820: tráfico portuario y consignatarios". En: Iglesias Rodríguez, Juan José (ed.): Andalucía en el mundo Atlántico Moderno: Agentes y escenarios, Sevilla: Silex, pp. 165-195.

Carrasco-González, Guadalupe (2017): "Importaciones y comercialización de los vinos del Marco de Jerez en Estados Unidos (1791-1824)”. En: Investigaciones de Historia Económica - Economic History Research, 13, 4, Madrid, pp. (en prensa).

Coggeshall, George (1856): History of the American privateers and letters of marque, during our war with England in the years 1812, 1813, 1814. Nueva York.

Coatswort, John C. (1967): "American Trade with European Colonies in the Caribbean and SouthnAmerica, 1790-1812". En: The William and Mary Quarterly, 24 (2), Williansburg, pp. 243-266.

Curtis, Charles B. (1883): Velázquez and Murillo: A descriptive and historical catalogue of the Works of Don Diego de Velázquez and Bartolomé Esteban Murillo, Nueva York.

Fernández Chaves, Manuel F/Gamero Rojas, Mercedes (2012): "El reino de Sevilla como escenario del ascenso del poder social y económico de los extranjeros en el siglo XVIII: el caso irlandés". En: Recio Morales, Oscar (ed.): Redes de nación y espacios de poder. La comunidad irlandesa en España y la América española, 1600-1825, Valencia: Albatros Ediciones-Ministerio de Defensa, pp. 191-217.

Fernández García, Ana María (1998-1999): "Pintura y comercio. Las relaciones anglo-españolas en el siglo XIX”. En: Norba, XVIII-XIX, Cáceres, pp. 31-241. 
García-Baquero González, Antonio (1972): Comercio colonial y Guerras revolucionarias. Sevilla: Escuela de Estudios Hispanoamericanos.

García González, Nélida (1999): Burguesía y toga en el Cádiz del siglo XVIII, Vicente Pulciani y su biblioteca ilustrada. Cádiz: Servicio de Publicaciones de la Universidad.

Gil-Díez Usandizaga, Ignacio (2011): "Ilustración y comercio. La biblioteca de Bernardo de Elías (1739-1791). Un Riojano en el comercio de Cádiz”. En: Berceo, 161, La Rioja, pp. 31-47.

Howat, John K/ Voorsanger, Catherine Hoover (eds.) (2000): Art and the Empire city New York, 1825-1861, New Haven, Londres: MET, Yale University Press.

Ibáñez Vergara, Jorge (1997): Don Nicolás de la Cruz, el Conde de Maule. Talca (Chile): Editorial Universidad de Talca. Kagan, Richard L. (2010): "The Spanish Craze in the United States: Cultural Entitlement and the Appropriation of Spain's Cultural Patrimony, 1890-1930". En: Revista Complutense de Historia, 36, Madrid, pp. 37-58.

Noah, Mordecai (1819): Travels in England, France, Spain and Barbary States in the year 1813-14 and 15. Nueva York: Publised by Kirk and Mercein.

Pemán Medina, María (1978): “La colección artística de Don Sebastián Martínez, amigo de Goya en Cádiz”. En: Archivo Español del Arte, 201, Madrid, pp. 53-62.

Ponz, Antonio (1794): Viaje de España, Madrid, XVIII.

Ravina Martín, Manuel (2001): "El mundo del libro en el Cádiz de la Ilustración”. En: Cuadernos de Ilustración y Romanticismo, 9, Cádiz, pp. 89-102.

Raynal Keene, Ricardo (1816): Memoria dirigida a S.M.C. el señor don Fernando VII sobre las calumnias del comerciante don Ricardo Meade importando alta traición contra los Estados Unidos y conspiración contra España, Madrid.

Reist, Inge/Colomer, José Luis (eds) (2012): Collecting Spanish Art: Spain’s Golden Age and America's Gilded Age. Nueva York: Frick Collection.

Roe, Jeremy (2010): "Customs Books and sales Catalogues: a Study of the importation of Spanish Painting into Britain". En: Glendinning, Nigel/ Macartney, Hilary (eds.): Spanish Art in Britain and Ireland, 1750-1820. Woodbridge: Tamesis, pp. 38-44.

Fecha de recepción: $30-X I I-2017$

Fecha de aceptación: 22-VI-2018 